\title{
NUMERICAL PREDICTION OF DISPERSION CHARACTERISTICS IN AN URBAN AREA BASED ON GRID REFINEMENT AND VARIOUS TURBULENCE MODELS
}

\author{
G. THEODORIDIS ${ }^{1 *}$, V. KARAGIANNIS ${ }^{2}$ and D. VALOUGEORGIS ${ }^{2}$ \\ ${ }^{1}$ AIAS Research Ltd, Thessaloniki, Greece; ${ }^{2}$ Department of Mechanical and Industrial \\ Engineering, University of Thessaly, Volos, Greece \\ (* author for correspondence, e-mail: georgios@aias.com.gr, fax: +30 310402060)
}

\begin{abstract}
A detailed simulation of the Goettinger Strasse pollutant dispersion problem is performed using the CFD code CFX-TASCflow for different wind directions. Two turbulence models, the $k-\varepsilon$ and the RSM are adopted on three grid refinement levels. Besides the typical reference grid implemented by the TRAPOS group, two different grid resolutions are introduced. The first refinement is in the whole street canyon region on the $\mathrm{x}-\mathrm{y}$ level, while the second one is local in all three directions. Validation of all involved computational schemes is performed based on relative available experimental data. The computed velocity fields and concentration contours imply that the typical reference grid is a suitable choice for the velocity fields, while local grid refinement in all three directions in a small region containing the receptor is required to upgrade the pollutant concentration results with modest additional computational effort. Finally the RSM model resulted in smaller concentration levels. The $k-\varepsilon$ model compared to the RSM seems a more appropriate choice to solve this particular problem.
\end{abstract}

Keywords: CFD, grid dependence, turbulence modeling, urban air quality

\section{Introduction}

The dispersion of pollutants in urban scale is dominated by modifications of the atmospheric flow caused by buildings. Down-wash phenomena and increased local turbulence strongly influence the mean flow field and the diffusion parameters. Early studies (Yamartino and Wiegard, 1986; Depaul and Sheih, 1986) with simple urban canyons, subject to perpendicular flow, have provided some description of the mean flow recirculation pattern. The influence of the aspect ratio (width/height) on the main vortex has been examined and it has been found that it is shifting upwards with decreasing canyon width (Depaul and Sheih, 1986), resulting into significantly higher pollutant concentrations in canyons with low aspect ratios (Pavageau et al., 1996). Further experimental studies have investigated the influence of different roof shapes on the distribution of pollutants (Rafailidis, 1997; KastnerKlein and Plate, 1999). The effect of different wind tunnel geometrical resolution and details on the dispersion of pollutants, has been examined by Liedtke et al. (1999) and Leitl et al. (2001). They found significant differences depending upon 
the complexity and the details of the experimental model. In addition they have noticed large discrepancies comparing their results with numerical simulations (Berkowitcz et al., 1997; Ketzel et al., 1999).

It has become common evidence that the observed changes in flow patterns and pollutant concentrations highly depend on the detailed geometry of the street canyon (Scaperdas, 2000). It is necessary to introduce the so-called obstacle resolving or microscale models. Although a wide variety of such models have been recently developed still further calibration and validation is needed (Schatzmann et al., 1999). The introduced uncertainties include typical computational problems (e.g. truncation error of the numerical scheme, implementation of boundary conditions, grid resolution, etc.). More specific difficulties are related with turbulence modeling and dispersion of pollutants in regions with high concentration gradients. Most of the existing studies are based on the typical $k-\varepsilon$ model (Sini et al., 1996), which seems to be adequate when isolated buildings or simplified street canyons are simulated. When the street canyon becomes more complex it has been suggested by Kim and Boysan (1999) that more advanced models such as the RSM (Launder et al., 1975; Speziale et al., 1991) must be implemented. In addition Schatzmann et al. (1999) claim that special attention is needed in areas with high concentration gradients such as the regions close to the sources. As a result all implemented computational models must be carefully tested against reliable experimental data, before their application to real practical situations.

Recognising the importance of model validation for solving pollutant dispersion problems in urban areas, the computational group of the DGXII-TMR, TRAPOS project initiated and executed a series of benchmarking exercises, which included comparisons between experimental and numerical results. The most complex among them is the Goettinger Strasse case, in the city of Hanover. For this particular problem, field measurements (NLÖ, 1995) and laboratory data (Liedtke and Schatzmann, 1999) were available for pollutant concentration at one receptor point close to the traffic pollution sources and for meteorological data over the roof of the highest building. Very recently, Chauvet et al. (2001) have performed additional measurements for the same configuration providing additional data for wind velocity within the street canyon. They are also stressing out the need for comprehensive wind-tunnel data under carefully controlled conditions, before numerical models could be validated. The Goettinger Strasse case has also been simulated numerically with the aid of various microscale models by Ketzel et al. (2001) using one relatively coarse grid and the standard $k-\varepsilon$ model.

In this article an attempt is made to investigate some of the above issues and to provide specific answers. In particular the well-known and commonly used $k-\varepsilon$ model is compared to the more advanced and time demanding Reynolds Stress transport Model (RSM) in order to examine the influence of turbulence anisotropy to the dispersion characteristics in urban areas. Moreover several grid resolutions are implemented to study the effect of grid refinement on the produced velocity and concentration fields. Schatzmann et al. (1999), claimed that, when the receptor 
and source-containing-cells are adjacent, the concentration results at the receptor are strongly grid dependent. For that reason, besides the typical reference grid implemented by the TRAPOS group, two different grid resolutions are introduced. The first refinement is in the whole street canyon region on the $\mathrm{x}-\mathrm{y}$ level, while the second one is local in all three directions.

\section{Computational Methodology}

Computational work is based on the CFX-TASCflow package, which is a general purpose CFD analysis system using a flexible multi-block curvilinear grid system. Within CFX-TASCflow, the conservation equations for mass, momentum, and scalar quantities like temperature, turbulent kinetic energy and any number of species are solved in curvilinear co-ordinates. The numerical solution is based on second-order in time and space discretisation, applied on a co-located grid arrangement. The discrete momentum and continuity equations are solved with a coupled elliptic solver. An efficient algebraic multi-grid solution technique is adopted, giving a practically constant rate of convergence, regardless the level of the grid refinement. A detailed description of the computational method can be found in Raw et al. (1989).

Turbulent diffusion can be described with the standard two-equation $k-\varepsilon$ turbulence, or the RSM, which unlike the standard $k-\varepsilon$ accounts for anisotropy of turbulence by solving six additional transport equations for the Reynolds stresses. Both models use the wall function approach to model near-wall viscous effects (Launder and Spalding, 1974). This approach assumes the universality of a logarithmic velocity profile in the near wall region and relies on the validity of near-wall turbulent equilibrium.

\section{Grid Resolution and Boundary Conditions}

The test case considered in this study corresponds to a complex urban area located in the Goettinger Strasse in Hanover. Laboratory data are available for pollutant concentration at one receptor location (see Figure 1) and vertical wind velocity and turbulence kinetic energy profiles over the anemometer (Liedtke and Schatzmann, 1999). The computational model in the present work is an exact replica of the experimental set up. The specifications of the case under consideration, including inflow boundary conditions and aerodynamic roughness, have been obtained from the www database (http://www.dmu.dk/AtmosphericEnvironment/trapos/cfd-wg.htm) of the DGXII-TMR TRAPOS project. Given a wind direction $\phi$, the corresponding Dirichlet inflow boundary conditions are applied at two of the four lateral bound- 


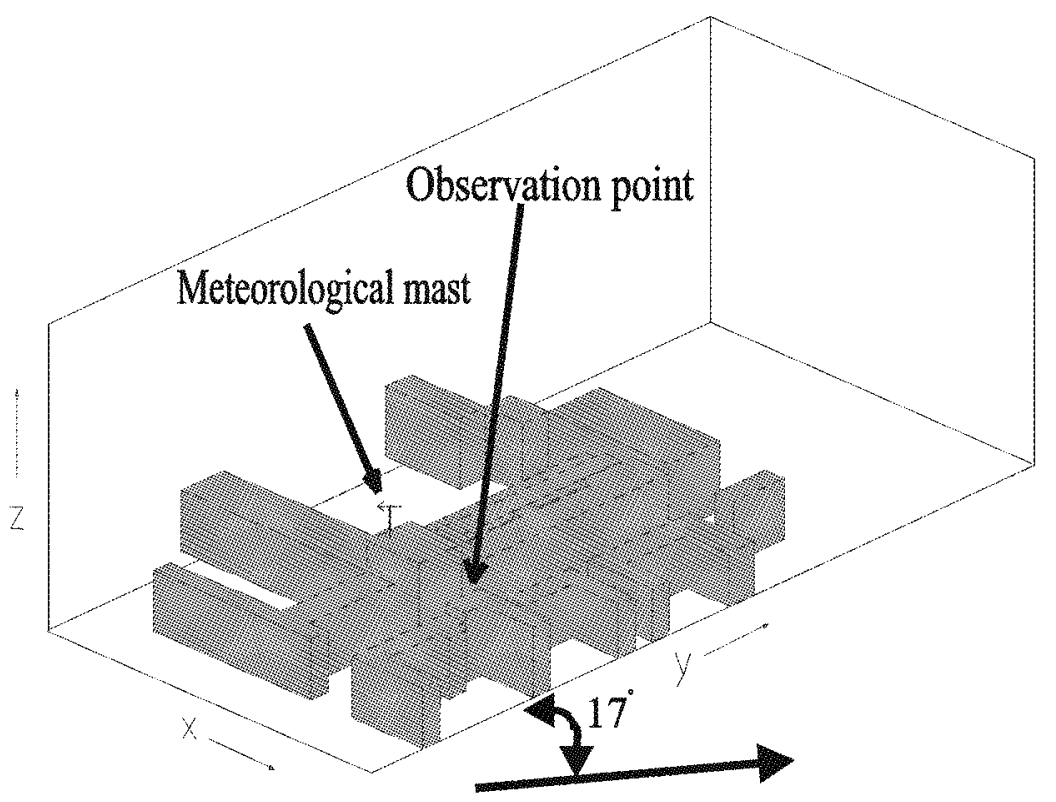

Figure 1. Geometrical configuration of urban area.

aries, while typical outflow conditions are applied at the remaining two. More specifically at the inflow boundaries the relationships

$$
\frac{V}{u_{*}}=\frac{1}{\kappa} \ln \left(\frac{z}{z_{0}}\right), \quad k=\frac{u_{*}}{\sqrt{c_{\mu}}}, \quad \varepsilon=\frac{u_{*}}{\kappa z},
$$

are used, where $V$ is the horizontal wind speed, $z$ is the distance from the ground, $u_{*}=0.526 \mathrm{~m} \mathrm{~s}^{-1}$ is the friction velocity, $z_{0}=0.05 \mathrm{~m}$ is the aerodynamic roughness and the empirical constants $\kappa, c_{\mu}$ take the values of 0.41 and 0.09 , respectively. All walls are considered as rough no-slip walls with an aerodynamic roughness of $z_{0}=0.01 \mathrm{~m}$. At the upper boundary a symmetry condition of vanishing gradients with respect to $z$ and vanishing normal velocity component $w$ is applied. To justify the choice of the symmetry condition the distance between the lower and upper boundaries is taken equal to twelve times the average building height. Appropriate sources with a total strength of $Q\left(\mathrm{Vol} \mathrm{s}^{-1}\right)$ are applied within the Goettinger Strasse, simulating the four-lane traffic emissions as shown in Figure 2.

Initially numerical simulations are performed on the reference numerical grid of $60 \times 73 \times 25$ points proposed by the CFD working group of the DGXII-TMR TRAPOS project (see Figure 2). Then a grid refinement (grid embedding) is applied in the $\mathrm{x}$ and $\mathrm{y}$ directions in the area bounded by the dashed frame, up to the roof of the highest building $(z=30 \mathrm{~m})$, as shown in Figure 2. It is expected that since the receptor is located very close to the sources, the whole area adjacent to the sources and the receptor is a high concentration gradient region. By tripling the 


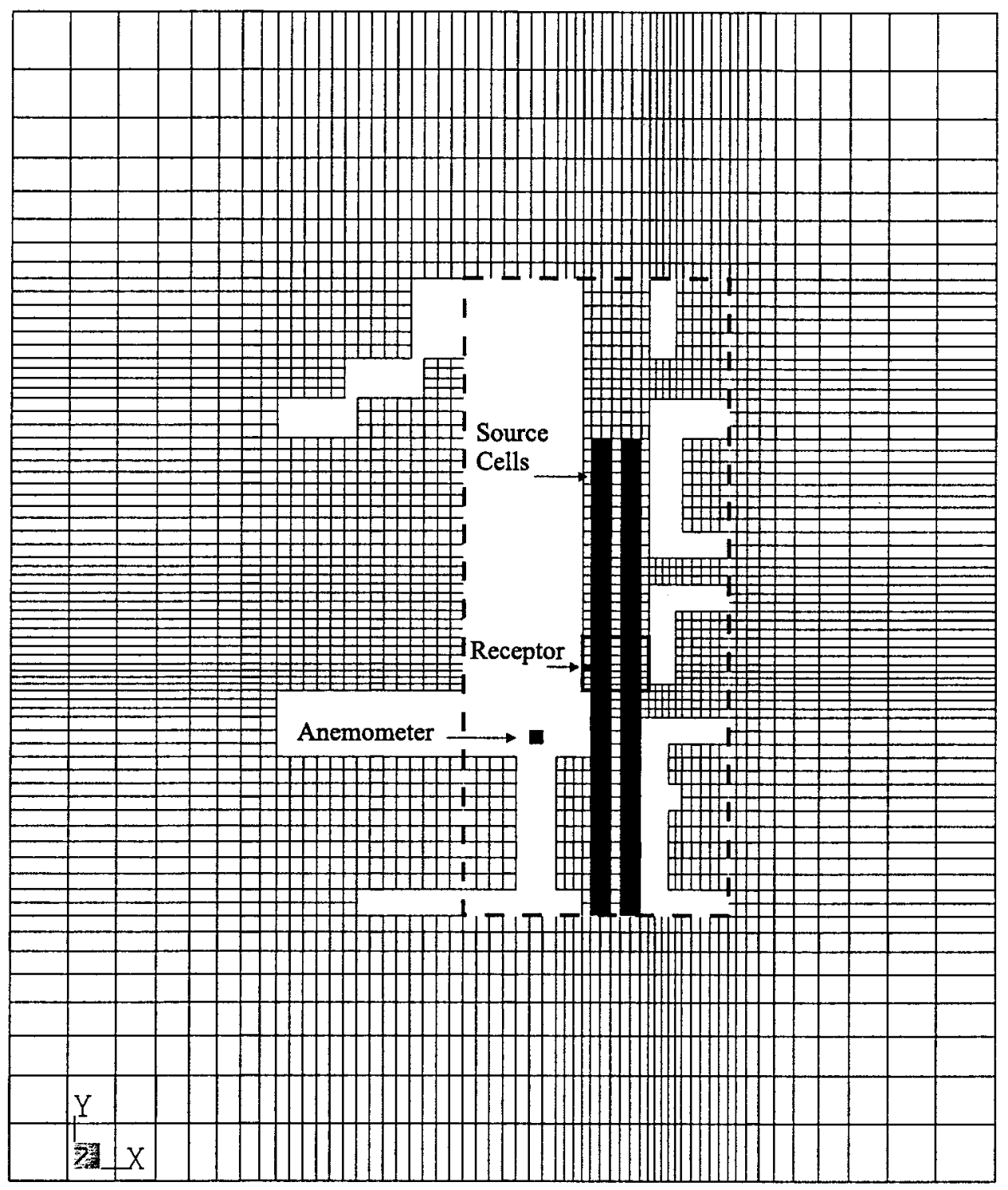

Figure 2. Reference grid and embedding regions at ground level. The grid is also refined by a factor of three in the horizontal direction (dashed line) and in all three directions close to the sensor location (solid line). 
number of nodes in the $\mathrm{x}$ and $\mathrm{y}$ directions the relative coarse set up of the reference grid is refined and the grid dependency of the velocity and concentration fields is studied. This yields $82 \times 169 \times 12$ additional grid points in the refined region. Finally in order to examine the influence of the volume of the source-containingcells on the computed concentration profiles (Schatzmann et al., 1999), the near receptor region (area bounded by the blue frame in Figure 2 until the height of $z=$ $17.5 \mathrm{~m}$ ) is refined in all three directions. As a result $21 \times 21 \times 21$ grid points are added to the reference grid.

The computed concentration field $c(\mathrm{Vol} / \mathrm{Vol})$ is non-dimensionalised using the relationship $c^{*}=\frac{c V_{\text {ref }} H}{Q / L}$, where $V_{\text {ref }}=10 \mathrm{~m} \mathrm{~s}^{-1}$ is the reference wind speed velocity at a height of $100 \mathrm{~m}, H=20 \mathrm{~m}$ is the average building height, and $L$ $=180 \mathrm{~m}$ is the length of the line sources.

\section{Results and Discussion}

In this section first a comparison of our numerical results with experimental data is attempted and then the detailed velocity and concentration fields are given at selected positions within the street canyon, based on all three employed grid resolutions and two turbulence models. It is well known that, depending on the wind direction, minor geometrical modifications may result to significant changes in pollutant concentrations. Thus the validation of the present numerical work is based on the experimental data obtained from the simplified structure of the wind tunnel model with closed gateway wing (Schatzmann et al., 1999). The geometric configuration of our virtual model, as mentioned before, is an exact replica of this experimental set up.

A first comparison between computed and measured velocity components and turbulence kinetic energy in the region above the anemometer is given in Figure 3 , for two characteristic wind directions $\left(\phi=0^{\circ}\right.$ and $\left.\phi=180^{\circ}\right)$. The computational results are obtained using the reference grid and both turbulence models under investigation. As it is seen the $u$ and $w$ profiles are slightly underestimated and overestimated respectively, while the $v$ profile is in excellent agreement compared to the measured corresponding quantities for both turbulence models. The computed turbulent kinetic energy curves match perfectly the measured ones for the $k-\varepsilon$ turbulence model, while the RSM based results are slightly underdetermined. In the region however, very close to the roof of the highest building, where the experimental data show high levels of $k$ there is an underestimation of the computed results for both models. Based on these results of the anemometer area, it seems that the $k-\varepsilon$ model produces superior results compared to the RSM results. Of course the overall agreement between computed and measured quantities is encouraging and remains unchanged for all wind directions tested between $0^{\circ}$ and $360^{\circ}$ with intervals of $10^{\circ}$. 

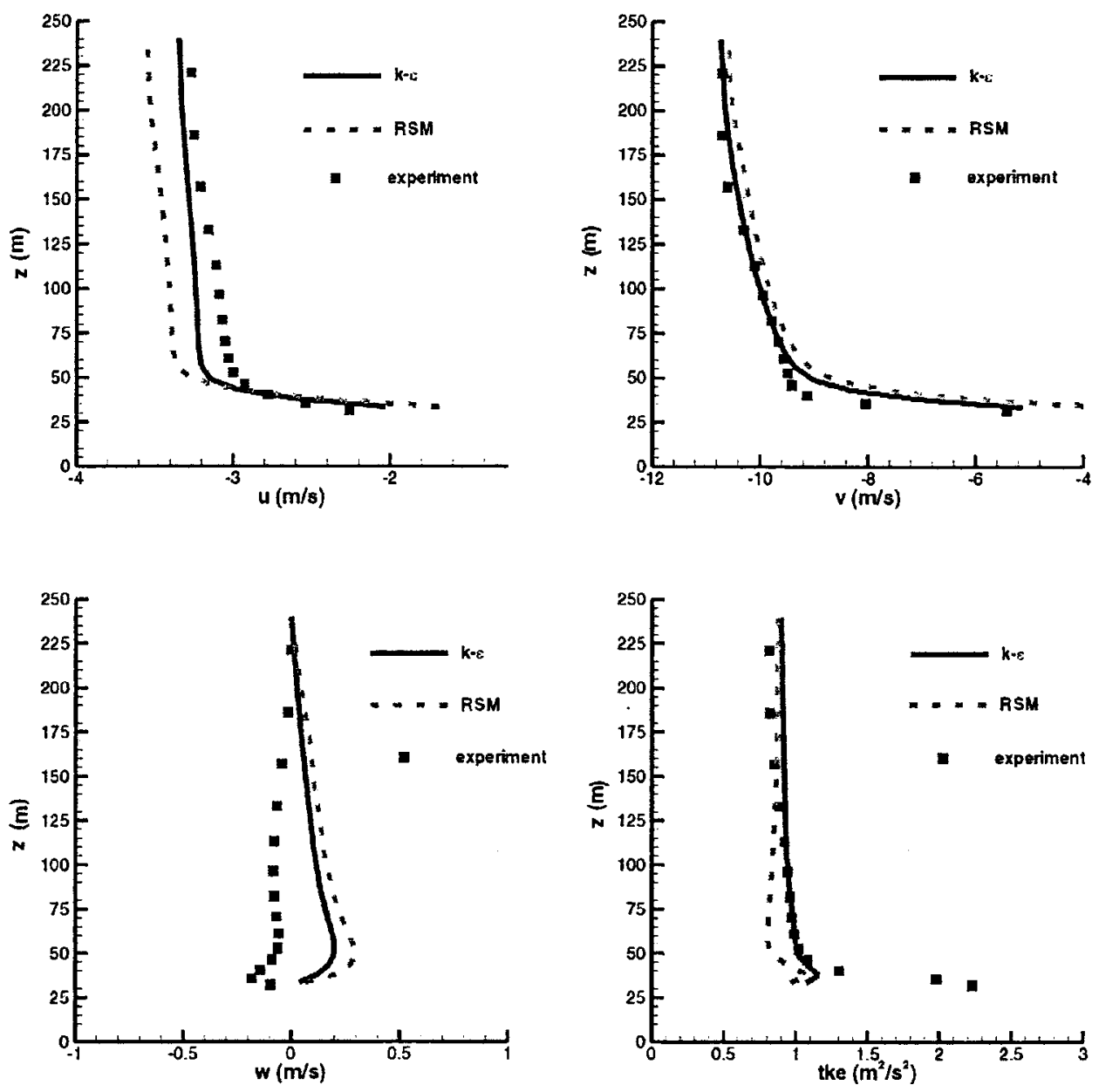

Figure $3 a$. Comparison between computed and measured wind components and turbulence kinetic energy at the region above the anemometer, for $\phi=0^{\circ}$.

Next in Figure 4 the computed concentrations at the monitoring point are compared to the corresponding measured ones for the complete range of the wind impingement angle. The results based on the $k-\varepsilon$ model are given for all three grid resolutions under consideration (i.e. the reference, the horizontaly refined and the grid with local refinement in all three directions), while the RSM results are shown only for the reference grid. It is seen that the shape and the peaks of the wind tunnel curve are reproduced and in most cases good qualitative and quantitative agreement between computational results and measurements is achieved. It is also obvious however that in certain wind direction angles the agreement is poor. In particular for $\phi=100 \pm 10^{\circ}$ and $\phi=220 \pm 10^{\circ}$ the departure between computed and measured concentrations is significant. This discrepancy may be due to the 

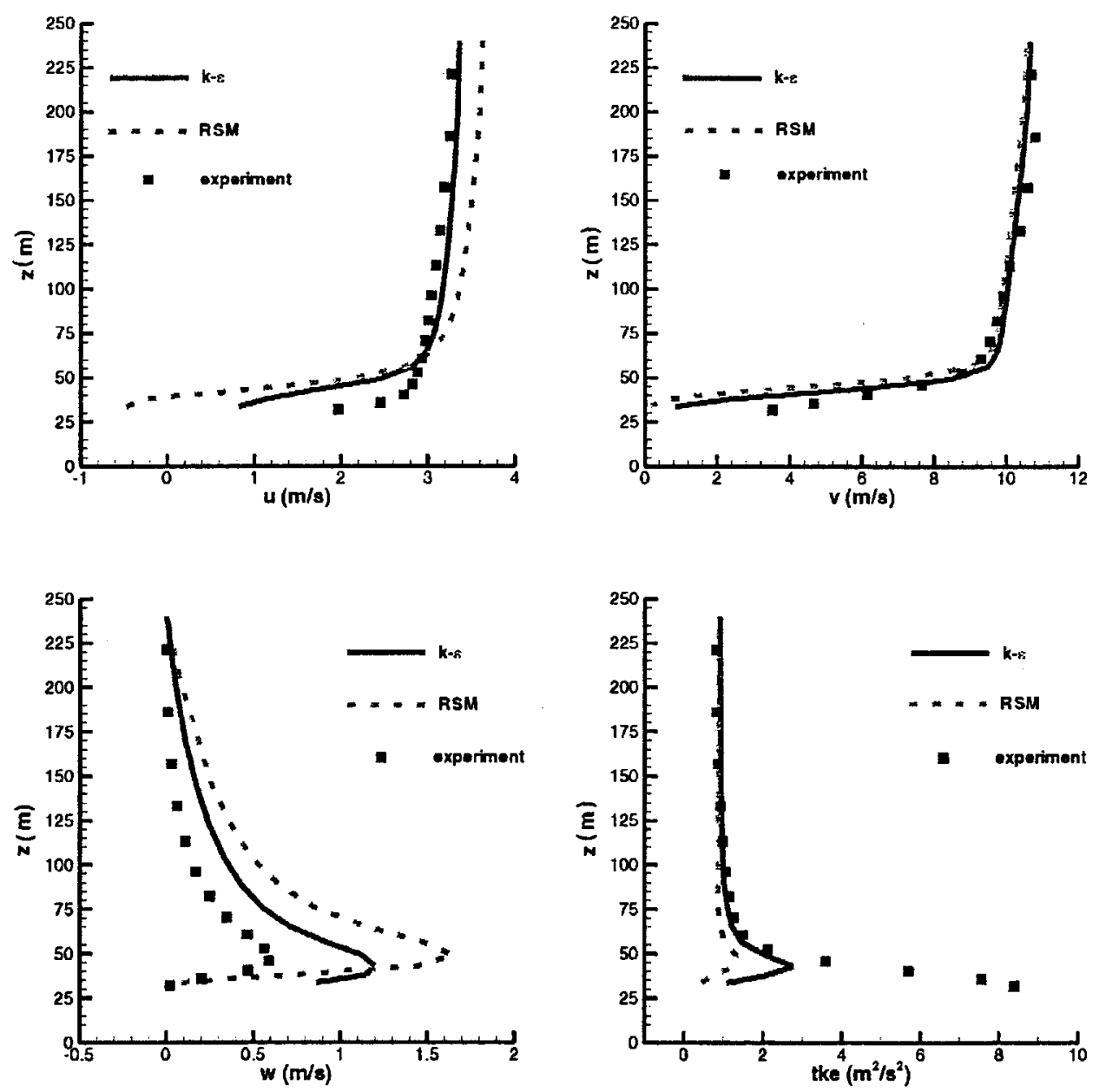

Figure $3 b$. Comparison between computed and measured wind components and turbulence kinetic energy at the region above the anemometer, for $\phi=180^{\circ}$.

fact that in the aforementioned wind directions the area around the receptor is characterised by high concentration gradients. As a consequence the uncertainties for both numerical and experimental work are increased. For example, small probe placement errors in the experiments would result in different probe readings. As reported earlier by Schatzmann et al. (1999) error bars of the order of $\pm 30 \%$, due to the size of the averaging time interval, should be considered in the particular wind tunnel results.

The results between the reference and the horizontal grid refinement are very close to each other (relative differences are less than 20\%) indicating that no significant improvement is achieved by refining the grid in the horizontal direction only. It is emphasised that in this case the additional computational effort and required 


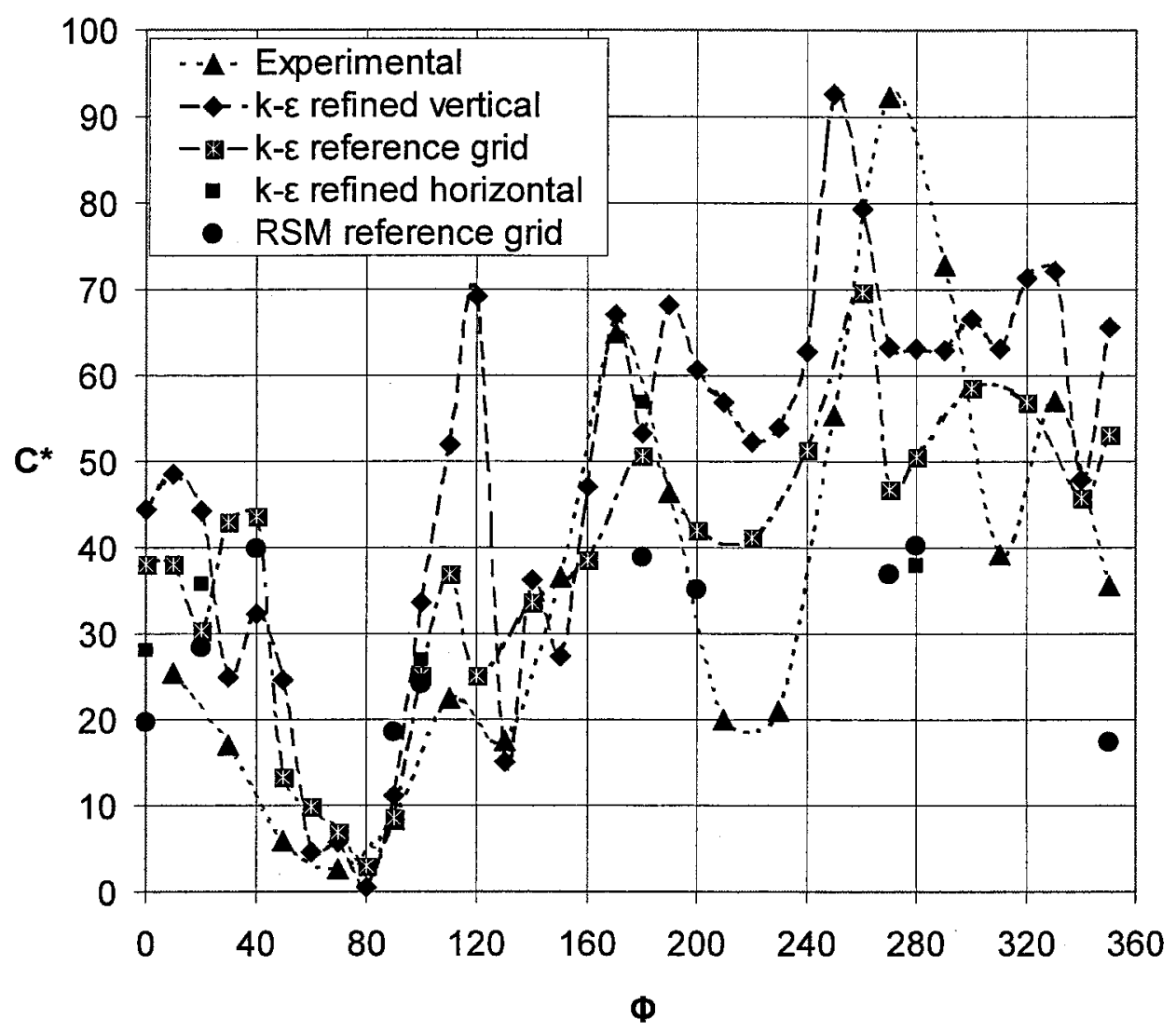

Figure 4. Comparison of the computed non-dimensional concentrations at the monitoring point for both turbulence models and all computational grids with experimental data.

memory space are both increased by a factor 4 . On the other hand a properly selected local refinement of the grid in all three directions leads to quite different computed receptor concentrations. These concentrations compared with the ones obtained with the other two computational grids are overall in better agreement with the measured concentrations. This is not the case again for the specific wind directions with $\phi=120^{\circ}$ and $\phi=220^{\circ}$, where the measured concentrations are overestimated by a factor of about three. As mentioned before there is some significant difference between computed results with the different cell sizes but this departure is not proportional to the refinement of the source grid cells as it is speculated in previous work (Schatzmann et al., 1999). This is an interesting matter, which will be discussed later in the article when the complete concentration profiles are given. Also in the same Figure 4, it is shown that the concentrations obtained with the RSM model and the reference grid are very close to the corresponding concentrations obtained with the $k-\varepsilon$ model. This is an indication that, at least for this particular problem under investigation, implementation of the RSM, which in 
general is a more advanced and reliable model than the $k-\varepsilon$, does not lead to more accurate results. Probably large eddy simulation models, able to directly simulate the large structure of atmospheric turbulence, may be the only remaining option when the required computational power is available. Finally it is noticed that in the local grid refinement case the required computational time and space compared to the corresponding ones with the reference grid are increased only about $20 \%$. At this stage it seems appropriate to notice that in order to validate further the existing numerical models in an accurate and consistent manner more experimental data are required. In order to propose with confidence the best suited model for the particular problem it is essential to have complete experimental sets of data instead of the presently available isolated experimental values at one or more locations.

We turn now our attention to the detailed velocity and concentration prediction within the street canyon. In all following work the presented results are for $\phi=0^{\circ}$ and they are representative for all other wind directions. In Figure 5 the horizontal velocity vector fields are shown at $z=3 \mathrm{~m}$ for the $k-\varepsilon$ on the reference and the horizontally refined grids (left and middle panels, respectively) and the RSM model on the reference grid (right panel). For reasons of clarity, velocity vectors are only plotted every second grid location in both $\mathrm{x}$ and $\mathrm{y}$ directions. It can be seen that the $k-\varepsilon$ results on both grids correspond to very similar flow patterns. It is noticed that although the wind direction is almost parallel to the longitudinal axis of the main street, the flow is not channeling inside the Goettinger Strasse, due to the fact that wind is coming also from the three normal streets on the right side of the street canyon. Actually several recirculation regions are developed in both the reference and the horizontally refined grids. This is clearly indicated at the center panel of Figure 5. It is concluded that the reference grid is a suitable grid with grid independent numerical results for the velocity fields. This statement is valid only when second order accurate schemes are adopted for the advection terms, as in the case of the CFX-TASCflow. The wind field results inside the street canyon for the $k-\varepsilon$ and the RSM are quite similar, indicating that there is no justification to select a more complex and time consuming turbulence model, such as the RSM, over a conventional type model, such as the $k-\varepsilon$. This conclusion remains invariant for all wind impingement angles tested. As a result the RSM has not been tested any further with the other two grids implemented in the present work.

As stated by Schatzmann et al. (1999) in most numerical models the initial concentration (the concentration in the source containing grid cells) is solely dependent on the actual choice of the grid due to the fact that the pollutant flux is uniformly distributed over the source grid cells. Thus decreasing the volume of these source grid cells by a certain factor (or in other words increasing accordingly the grid resolution in the $\mathrm{z}$ direction) results into an increase of the initial concentration by the same factor. As a consequence, it is expected that the concentration of the grid cells adjacent to the source will be controlled, to a large degree, by the actual choice of the grid dimension. 

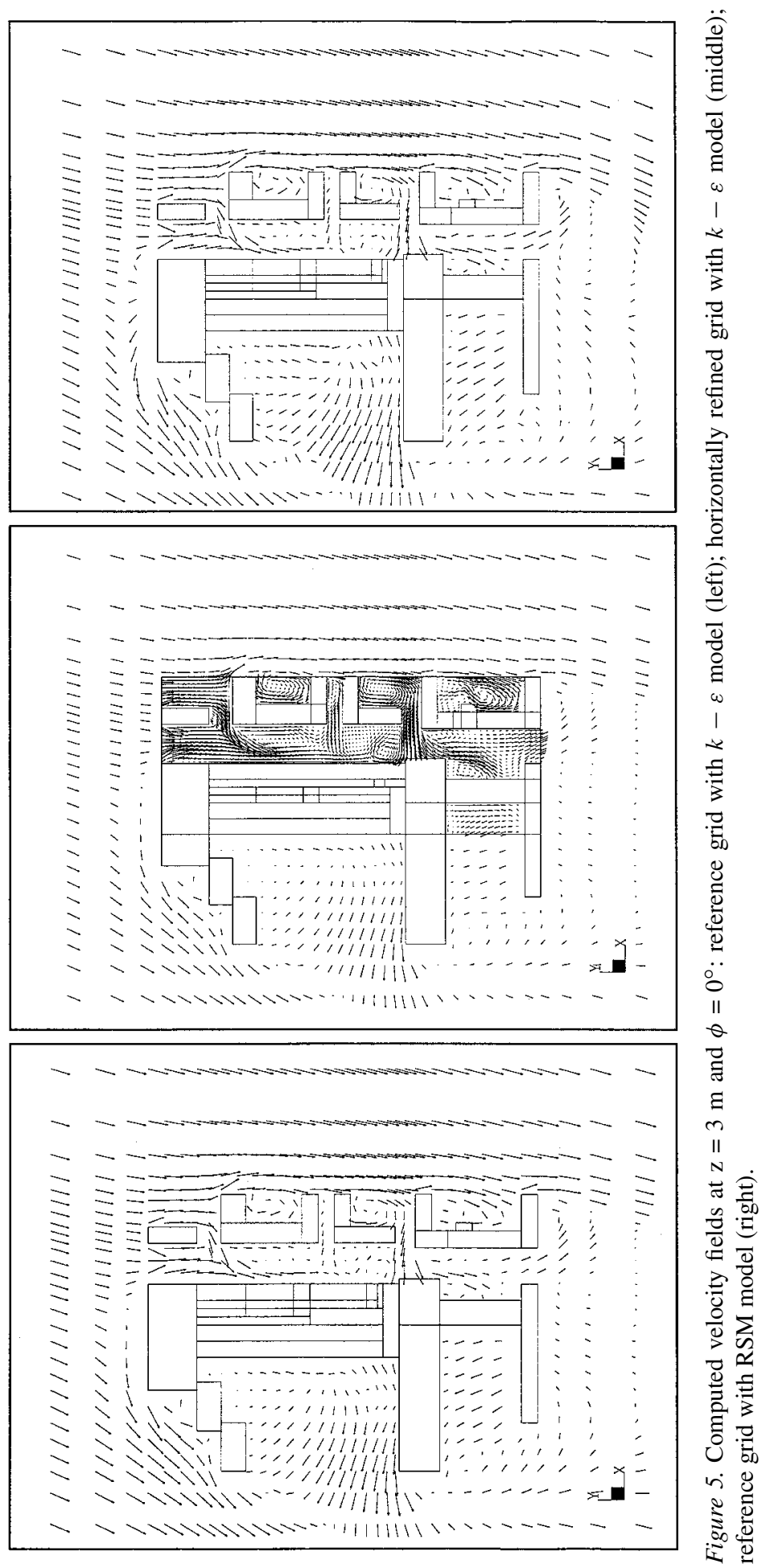

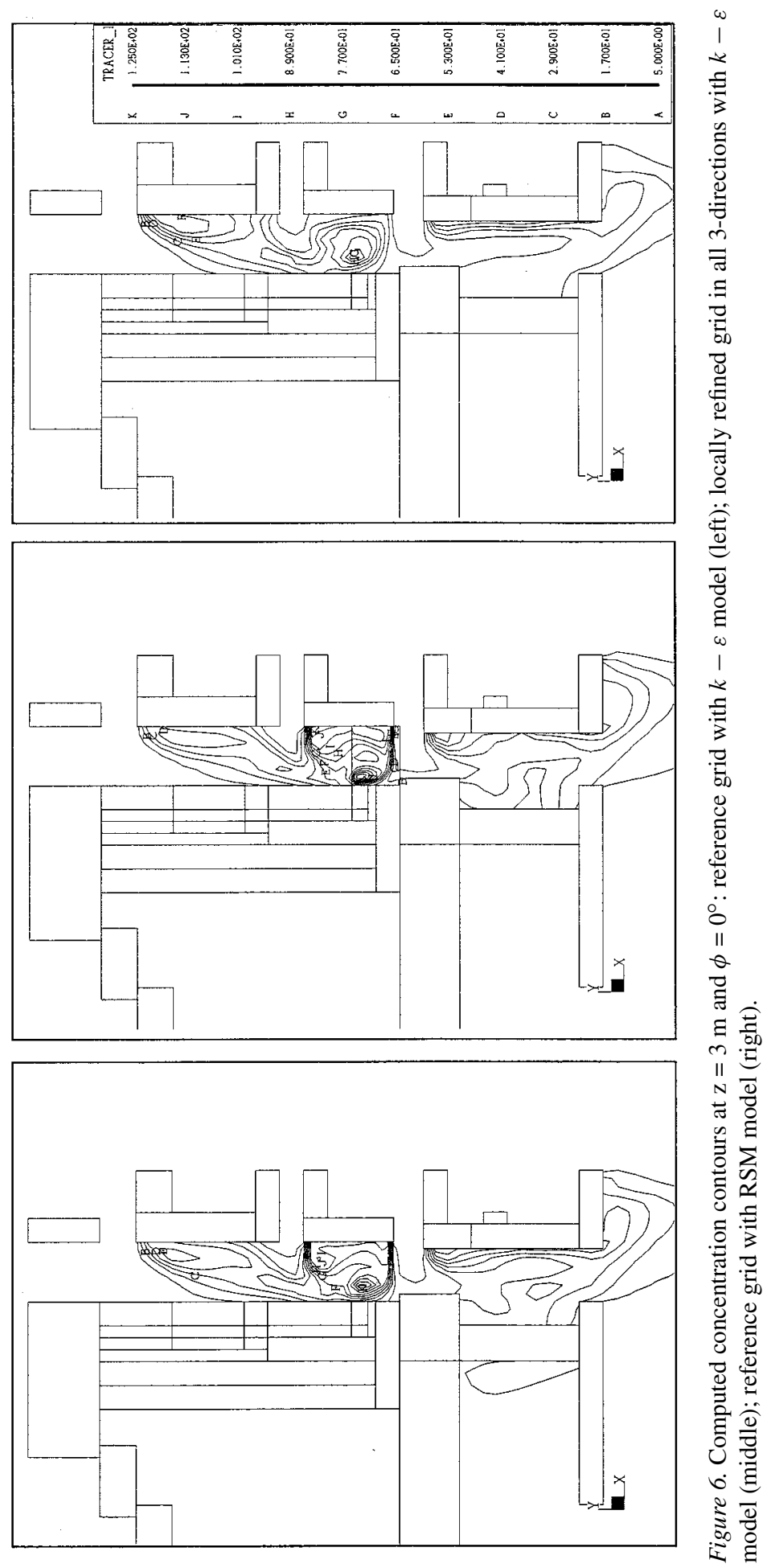
As mentioned before the wind field is found to be insensitive to the horizontal grid refinement. Therefore the above interesting concern is tested by adopting a local grid refinement in all three directions only in the high gradient concentration region close to the receptor (see Figure 2). In the resulting grid, produced by tripling the number of points locally, there is one and a half-cell between the receptor and the sources on both the $\mathrm{x}$ and $\mathrm{z}$ directions. It is emphasised that in previous work by other investigators, related to the Goettinger Strasse, the receptor was always placed at the center of the first boundary cell adjacent to the source street lines. In Figure 6 the concentration contours are indicated for the $k-\varepsilon$ model on the reference and the locally refined grids (left and middle panels), as well as the RSM model on the reference grid (right panel). These concentration fields correspond to two of the velocity vector fields shown in Figure 5 (left and right panels). As it is seen the results with the standard $k-\varepsilon$ including the horizontally refined grid case which is not shown here, are quite similar. Maximum concentrations as high as $c^{*}=125$ are experienced, located in the aforementioned regions of recirculating flow (see also Figure 5). More important, although the initial concentration at the source-containing-cells has been increased by a factor of three the corresponding receptor concentration has been increased, compared to that of the reference grid, only by $17 \%$ (see Figure 4). It is concluded that at the height of $z \geq 3 \mathrm{~m}$, the numerical results are practically grid independent. The concentration results at the receptor however are more sensible when local grid refinement in all three directions is applied.

Finally the concentration field, which is based on the reference grid and the RSM is shown in the right panel of Figure 6. Although the velocity fields inside the street canyon region are very similar for the two turbulence models, it seems that the RSM model tends to overpredict the eddy diffusivity, resulting in an overall underestimation of the concentration of pollutants. It is noticed that the $k-\varepsilon$ results based for the reference grid are in excellent agreement with recent computational results by Ketzel et al. (2001). As far as the authors are aware of there no available results in the literature to compare with for the case of the RSM and more dense grid resolutions.

\section{Conclusions}

A detailed simulation of the Goettinger Strasse pollutant dispersion problem is performed using the CFD code CFX-TASCflow for different wind directions. Two turbulence models, the $k-\varepsilon$ and the RSM are adopted and three grid refinement levels are used. Validation of all involved computational schemes is performed based on relative available experimental data. Complete velocity vector fields and concentration contours are reported.

The velocity fields are found to be similar for all implemented grids and turbulence models. This implies that the so-called 'reference grid' is a suitable choice 
with grid independent numerical results for the velocity fields, when second order accurate schemes are adopted for the advection terms. From the other hand, the so-called 'local refined' grid in all three directions in the region containing the receptor is the most suitable way to upgrade the pollutant concentration results with modest additional computational effort. Finally the RSM model resulted in smaller concentration levels. The $k-\varepsilon$ model seems a more appropriate choice to solve this particular problem compared to the RSM. Although the above conclusions are related to the specific problem under consideration general guidelines may be obtained, since the present problem is the most complex one in a series of test cases performed for model validation (Ketzel et al., 2001).

It is important to notice that at this stage of the investigation is essential to have complete experimental sets of data instead of the presently available isolated experimental values at a very limited number of locations. Assuming that these experimental results are available the present work may be extended to validate existing computational models in a more integrated manner.

\section{Acknowledgements}

The specifications of the case studied were prepared by the CFD working group of the DGXII-TMR TRAPOS project. Availability of the specification and measurement data is highly acknowledged.

\section{References}

Berkowicz, R., Hertel, O., Larsen, S. E., Sorensen, N. N. and Nielsen, M.: 1997, Modelling Traffic Pollution in Streets, National Environment Research Institute, Roskilde, Denmark.

Chauvet, C., Leitl, B. and Schatzmann, M.: 2001, 'High Resolution Measurements in an Idealised Street Canyon', The 3rd International Conference on Urban Air Quality, Loutraki, Greece, March 2001.

DePaul, F. T. and Sheih, C. M.: 1986, 'Measurements of wind velocities in a street canyon', Atmosph. Environ. 20, 555-559.

Kastner-Klein, P. and Plate, E. J.: 1999, 'Wind tunnel study of concentration fields in street canyon', Atmosph. Environ. 33, 3973-3979.

Ketzel, M., Berkowicz, R. and Lohmeyer, A.: 'Dispersion of Traffic Emissions in Street Canyons - Comparison of European Numerical Models with Each Other as Well as with Results from Wind Tunnel and Field Measurements', The 3rd International Conference on Urban Air Quality, Madrid, March 1999.

Ketzel, M., Louka, P. Sahm, P. Guilloteau, E., Sini, J. F. and Moussiopoulos, N.: 2001, 'Intercomparison of Numerical Urban Dispersion Models - Part II: Street Canyon in Hanover, Germany', The 3rd International Conference on Urban Air Quality, Loutraki, Greece, March 2001.

Kim, S. E. and Boysan F.: 1999, 'Application of CFD to environmental flows', J. Wind. Eng. 81, $145-158$.

Launder, B. E., and Spalding, D. B.: 1974, 'The numerical computation of turbulent flows', Comput. Meths. Appl. Mech. Eng. 3, 269-289. 
Launder, B. E., Reece, G. J. and Rodi, W.: 1975, 'Progress in the development of a Reynolds-stress turbulence closure', J. Fluid Mech. 68, 537-566.

Leitl, B., Chauvet, C. and Schatzmann, M.: 2001, 'Effects of Geometrical Simplifications and Idealization on the Accuracy of Microscale Dispersion Modeling', 3rd International Conference on Urban Air Quality, Loutraki, Greece, March 2001.

Liedtke, J. and Schatzmann, M.: 1999, 'Autoabgasausbreitung in Straßenschluchten - Vergleich von Windkanal- und Naturmessungen an einem konkreten Fallbeispiel. Projekt Europäisches Forschungszentrum für Maßnahmen zur Luftreinhaltung. Forschungszentrum Karlsruhe', Schlußbericht PEF 296001 (in German).

Liedtke, J., Leitl, B. and Schatzmann, M.: 1999, 'Dispersion in a street canyon: Comparison of wind tunnel experiments with field measurements', in P. M. Borrel and P. Borrel (eds), Proc. of Eurotrac Symposium 98, WIT Press, Southampton 1999, pp. 806-810.

NLÖ: 1995, 'Lufthygienisches Überwachungssystem Niedersachsen-Standortbeschreibung der NLÖ Stationen', Bericht, Niedersächsisches Landesamt für Ökologie, Göttinger Strasse 14, 30449 Hannover (in German).

Pavageau, M., Rafailidis, S. and Schatzmann, M.: 1996, 'A Comprehensive Experimental Databank for the Verification of Urban Car Emission Dispersion Models', Proceedings of the 4th Workshop on Harmonisation within Atmospheric Dispersion Modeling for Regulatory Purposes, Ostende, May 1996.

Rafailidis, S.: 1997, 'Influence of building area density and roof shape on the wind characteristics above a town', Bound.-Layer Meteor. 85, 255-271.

Raw, M. J., Galpin, P. F. and Hutchinson, B. R.: 1989, 'A collocated finite-volume method for solving the Navier-Stokes equations for incompressible and compressible flows in turbomachinery: Results and applications', Canadian Aeronautics and Space Journal 35, 189-196.

Scaperdas, A.: 2000, 'Modelling Air Flow and Pollutant Dispersion at Urban Canyon Intersections', Ph.D. Thesis, University of London.

Schatzmann, M., Liedtke, J. and Leitl, B.: 1999, 'Dispersion Models for Urban Applications. A Critical Assessment of the Present State of Application', Internationale Kolloquium' Messung und Beurteilung der Luftqualität, Heidelberg, April 1999.

Sini, J. F., Anquetin, S. and Mestayer, P.: 1996, 'Pollutant dispersion and thermal effects in urban street canyons', Atmosph. Environ. 30, 15, 2659-2677.

Speziale, C. G., Sarkar, S. and Gatski, T. B.: 1991, 'Modeling the pressure-strain correlation of turbulence: An invarient dynamical systems approach', J. Fluid Mech. 227, 245-272.

Yamartino, R. J. and Wiegand, G.: 1986, 'Development and evaluation of simple models for the flow, turbulence and pollutant concentration fields within an urban street canyon', Atmosph. Environ. 20, 2137-2156. 